\title{
Expression of microRNAs in squamous cell carcinoma of human head and neck and the esophagus: miR-205 and miR-21 are specific markers for HNSCC and ESCC
}

\author{
SOTAI KIMURA $^{1,2}$, SEIJI NAGANUMA $^{1}$, DAI SUSUKI ${ }^{1,3}$, YASUO HIRONO $^{4}$, AKIO YAMAGUCHI ${ }^{4}$, \\ SHIGEHARU FUJIEDA ${ }^{3}$, KAZUO SANO ${ }^{2}$ and HIROSHI ITOH ${ }^{1}$ \\ ${ }^{1}$ Division of Tumor Pathology, Department of Pathological Sciences, Divisions of ${ }^{2}$ Dentistry and Oral Surgery \\ and ${ }^{3}$ Otorhinolaryngology Head and Neck Surgery, Department of Sensory and Locomotor Medicine, and \\ ${ }^{4}$ First Department of Surgery, Faculty of Medical Sciences, University of Fukui, Fukui, Japan
}

Received December 9, 2009; Accepted February 24, 2010

DOI: $10.3892 /$ or_00000804

\begin{abstract}
MicroRNAs (miRNAs) are non-coding small RNAs that regulate cell proliferation and functions by interfering with the translation of target mRNAs. Altered expression of miRNA is known to induce various human malignancies. We examined the expression of miRNAs in squamous cell carcinoma of human head and neck (HNSCC) and esophagus (ESCC), compared to that in normal squamous epithelia as well as malignancies of other organs. Microarray analysis showed up-regulation of miR-21, miR-16 and miR-30a-5p in HNSCC and ESCC cell lines compared to normal squamous epithelial cell lines, and consistent high expression of miR-205 and let-7a in both normal and malignant squamous epithelial cell lines. Validation study using real-time quantitative RT-PCR in formalin-fixed paraffin-embedded cancer tissues and paired normal epithelia obtained by Laser-captured microdissection revealed that miR-205 showed highest expression in both malignant and benign squamous epithelia, although it was less expressed in cell lines and tissues other than squamous epithelia. MiR-21, which is an oncogenic miRNA in various malignancies, was also up-regulated in HNSCC and ESCC compared to paired normal squamous epithelia. These results suggest that miR205 might be a specific marker miRNA of both normal and malignant squamous epithelia, while miR-21 might be a putative oncogenic miRNA in HNSCC and ESCC.
\end{abstract}

Correspondence to: Dr Hiroshi Itoh, Division of Tumor Pathology, Department of Pathological Sciences, Faculty of Medical Sciences, University of Fukui, 23-3 Matsuoka-Shimoaizuki, Eiheiji, Fukui 910-1193, Japan

E-mail: hiroitoh@u-fukui.ac.jp

Key words: head and neck, esophagus, microdissection, microRNA, squamous cell carcinoma

\section{Introduction}

Squamous cell carcinoma (SCC) is the most common malignant tumor in the head and neck region and the esophagus (1-3). While the incidence and mortality of the esophageal SCC (ESCC) has been gradually declining due to significant advances in surgery and radiotherapy over the last decades (1), those of head and neck SCC (HNSCC) is still increasing despite intense efforts, and its 5-year survival rates is less than $50 \%(2,3)$. To further improve outcomes of the patients with HNSCC and ESCC, novel diagnostic, therapeutic and prognostic biomarkers should be developed.

MicroRNAs (miRNAs) are non-coding small RNAs ( $\sim 22$ nucleotides) that regulate posttranscriptional gene expression by interfering with the translation of target mRNA $(4,5)$. One miRNA may regulate the expression of several genes and over one-third of all protein-coding genes may be under translational control by miRNAs (5). MiRNAs are involved in a variety of cellular processes, including the regulation of cellular differentiation, proliferation and apoptosis (5). Aberrant expression of miRNA is known to induce various human malignancies (6-9) and they are clearly classified by miRNA expression profiles $(9,10)$. MiR-21 functions as a putative oncogenic miRNA in brain and breast cancers by indirectly down-regulating the anti-apoptotic gene $B C L 2$, while miR-16 and let-7a act as tumor-suppressive miRNAs in B-cell lymphomas and colorectal carcinomas by directly up-regulating the translation of BCL2 and RAS oncogenes, respectively (6-9). MiR-205 was originally identified as an miRNA up-regulated in HNSCC cell lines (11).

In this study, we examined the expression of miRNAs in human HNSCC and ESCC, compared to those in normal squamous epithelia and malignancies of other organs. Two potential biomarker miRNAs for HNSCC and ESCC (miR-21) and squamous epithelia (miR-205) were identified by microarray analysis and following validation by real-time quantitative reverse transcription-polymerase chain reaction (qRT-PCR) using formalin-fixed paraffin-embedded (FFPE) tissue samples obtained by Laser-captured micro-dissection (LCM). We also discuss the application of miRNA qRT-PCR 
analysis for molecular diagnosis using routine FFPE histopathological tissue samples and LCM system, since miRNAs are very stable and could be successfully extracted from FFPE tissue samples stored over 20 years.

\section{Materials and methods}

Cell lines. Human tongue SCC cell lines SCC4, SCC9, SCC15 and SCC25 were purchased from the American Type Culture Collection (Rockville, MD, USA). Human tongue SCC cell lines OSC19 and OSC20, and human ESCC cell lines KYSE30, KYSE70, KYSE140, KYSE150, KYSE170, KYSE180, KYSE220 and KYSE270 were obtained from Japanese Collection of Research Bioresources (Osaka, Japan). Human tongue SCC cell lines HSC3, HSC4 and SAS, human hepatocellular carcinoma cell line HepG2, human colon cancer cell line LoVo, and human hematological cancer cell lines HL60, K562 and MOLT-4 were obtained from Riken cell bank (Tsukuba, Japan). Human colorectal cancer cell lines CoCM-1 and RCM-1:L10 were a kind gift of Professor H. Kataoka, University of Miyazaki, Japan. These cancer cell lines were cultured in Dulbecco's modified Eagle's medium (DMEM), HAM F-12 or RPMI-1640 media (Sigma-Aldrich, St. Louis, MO, USA), supplemented with $10 \%$ fetal bovine serum (FBS) (Nichirei Biosciences, Tokyo, Japan) and 1\% penicillin-streptomycin (Gibco Invitrogen, Carlsbad, CA, USA), according to the protocols of suppliers. Human normal oral keratinocyte cell line HOK and human normal esophageal sqaumous epithelial cell line HEEC were purchased from ScienCell (San Diego, CA, USA) and were grown in keratinocyte medium (OKM) and epithelial cell medium-2 (EpiCM-2) as recommended by the supplier, respectively. All of these cell lines were cultured at $37^{\circ} \mathrm{C}$ in a humidified atmosphere containing $5 \% \mathrm{CO}_{2}$.

Microarray analysis. Total RNAs from cultured cell lines were extracted by TRIzol ${ }^{\mathrm{TM}}$ reagent (Gibco Invitrogen) and quantified with NanoDrop ${ }^{\mathrm{TM}}$ spectrometer (NanoDrop Technology, Wilmington, DL, USA). The quality was checked by Agilent 2100 bioanalyzer (Agilent Technologies, Santa Clara, CA, USA), in order to assure that the RNA lacked DNA contamination and that the RNA was not degraded. MiRNAs were further purified from total RNAs by flash PAGE $^{\text {TM }}$ system (Applied Biosystems, Foster City, CA, USA) and labeled with mirVana ${ }^{\mathrm{TM}}$ miRNA Labeling kit (Applied Biosystems). MiRNA microarray analysis was performed by Filgen Inc. (Nagoya, Japan) using mirVana miRNA Bioarray V2 (Applied Biosystems), which contains 662 mature and pre-miRNAs including 328 human miRNAs. The arrays run were then scanned by GenePix ${ }^{\mathrm{TM}}$ 4000B scanner (Molecular Devices Corp., Sunnyvale, CA, USA) and their images were analyzed with Array-Pro ${ }^{\mathrm{TM}}$ Analyzer Ver.4.5 (Media Cybernetics Inc., Bethesda, MD, USA). Each microarray assay was performed three times using different sets of miRNA extracts and quantile normalization was performed.

Human tissue samples and their miRNA extraction. All human tissue samples were obtained from surgical specimens of patients with HNSCC, ESCC or other malignancies in the University of Fukui Hospital, Fukui, Japan. Informed consent was obtained from the patients before surgery and the protocol was approved by the ethics board of the Faculty of Medicine, University of Fukui. Tissue samples were taken from FFPE blocks of 13 HNSCC, 10 ESCC, 5 skin SCC, 5 cervical SCC, 5 lung SCC, 5 lung adenocarcinoma and 5 colorectal adenocarcinoma patients and from frozen sections of 7 patients with tonsillitis. Malignant and normal epithelia were separately obtained from these FFPE or frozen sections stained with Cresyl Violet Stain (Applied Biosystems) by LCM system (Leica Microsystems, Wetzler, Germany), according to the manufacturer's protocol. After pre-treatment of Proteinase K Solution (Invitrogen) at $55^{\circ} \mathrm{C}$ for $12 \mathrm{~h}$, their miRNAs were extracted by PureLink ${ }^{\mathrm{TM}}$ miRNA Isolation kit (Invitrogen) and quantified with NanoDrop spectrometer, described above.

Validation of miRNA microarray results by $q R T-P C R$. For validation of miRNA expression profiles in cultured cell lines, 13 HNSCC, 10 ESCC, 5 skin SCC, 5 cervical SCC, 5 lung SCC, 5 lung adenocarcinoma and 5 colorectal adenocarcinoma tissue samples were collected with paired normal epithelial tissue samples. Their miRNAs separately extracted by LCM system were subjected to quantitative 2-step RT-PCR as per the manufacturer's manual (Applied Biosystems). The kits used were TaqMan ${ }^{\circledR}$ MicroRNA Assay (for miR-16, miR-21, miR-205, let-7a and U6 small nuclear RNA as an inner control), TaqMan ${ }^{\circledR}$ MicroRNA Reverse Transcription kit and TaqMan ${ }^{\circledR}$ Universal PCR Master Mix (Applied Biosystems) for cultured cell lines and miScript Primer Assay (for miR-16, miR-21, miR-205, miR-30a-5p, let-7a and U6), miScript Reverse Transcription kit, and miScript SYBR $^{\circledR}$ Green PCR kit (Qiagen, Hilden, Germany) for human tissue samples. Real-time qRT-PCR was performed by StepOne ${ }^{\mathrm{TM}}$ Real-Time PCR System (Applied Biosystems). Reverse transcription was performed in the following conditions; $16^{\circ} \mathrm{C}$ for $30 \mathrm{~min}, 42^{\circ} \mathrm{C}$ for $30 \mathrm{~min}, 85^{\circ} \mathrm{C}$ for $5 \mathrm{~min}$ with a sample volume of $15 \mu \mathrm{l}$ (TaqMan MicroRNA Assays) or $37^{\circ} \mathrm{C}$ for $60 \mathrm{~min}$, then $95^{\circ} \mathrm{C}$ for $5 \mathrm{~min}$ with a sample volume of $20 \mu 1$ (miScript PCR System). The real-time PCR was performed as follows; $50^{\circ} \mathrm{C}$ for $2 \mathrm{~min}, 95^{\circ} \mathrm{C}$ for $10 \mathrm{~min}$, 40 cycles of $95^{\circ} \mathrm{C}$ for $15 \mathrm{sec}$ and $60^{\circ} \mathrm{C}$ for $1 \mathrm{~min}$ with a sample volume of $20 \mu \mathrm{l}$ (TaqMan MicroRNA Assays) or $95^{\circ} \mathrm{C}$ for $15 \mathrm{~min}, 40$ cycles of $94^{\circ} \mathrm{C}$ for $15 \mathrm{sec}$ and $55^{\circ} \mathrm{C}$ for $30 \mathrm{sec}$, and $70^{\circ} \mathrm{C}$ for $30 \mathrm{sec}$ with a sample volume of $20 \mu \mathrm{l}$ (miScript PCR System). The expression level was normalized to the U6 expression used as an inner control and was determined by the following processes; the average $\mathrm{C}_{t}$ of the inner control $\left(C_{t \text { inner }}\right)$ and the sample $C_{t}$ were determined. This value was then entered into this formula $=2^{\text {-(Ct-Ct inner) }}$. The average miRNA expression in normal tissue vs. malignant tissue was determined by the Mann-Whitney's U test using the MEDCALC software program (MedCalc software, Mariakerke, Belgium). A p-value $<0.05$ was considered to be significant. The values were expressed as the mean with standard errors (SE). To clarify whether miRNA was correctly amplified, RT-PCR products were separated with and cut from $4 \%$ agarose gel and the DNA was extracted and cloned into pCR II vector (Gibco Invitorogen). Nucleotide sequencing was performed using ABI PRISM 310 Genetic Analyzer (Applied Biosystems). 
Table I. The 12 highest expressions (net intensity) of miRNAs in malignant and benign squamous epithelial cell lines by miRNA microarray.

\begin{tabular}{|c|c|c|c|c|c|c|c|c|}
\hline & \multicolumn{2}{|c|}{6 HNSCC cell lines } & \multicolumn{2}{|c|}{$\mathrm{HOK}$} & \multicolumn{2}{|c|}{7 ESCC cell lines } & \multicolumn{2}{|c|}{ HEEC } \\
\hline 1 & miR-205 & 7024 & miR-205 & 5951 & $\operatorname{miR}-205$ & 8802 & miR-205 & 6065 \\
\hline 2 & miR-21 & 3552 & miR-31 & 2394 & miR-21 & 4173 & miR-31 & 4366 \\
\hline 3 & miR-24 & 2082 & miR-21 & 2054 & miR-16 & 2742 & miR-21 & 2851 \\
\hline 4 & miR-23a & 1818 & let-7a & 1817 & miR-200c & 2219 & let-7a & 1958 \\
\hline 5 & miR-31 & 1700 & $\operatorname{miR}-23 a$ & 1667 & miR-24 & 1783 & $\operatorname{miR}-23 b$ & 1571 \\
\hline 6 & let-7a & 1691 & miR-222 & 1543 & $\operatorname{miR}-23 b$ & 1663 & let-7c & 1557 \\
\hline 7 & miR-23b & 1442 & $\operatorname{miR}-23 b$ & 1667 & let-7a & 1639 & miR-23a & 1547 \\
\hline 8 & let- $7 b$ & 1393 & let-7c & 1339 & miR-23a & 1618 & miR-29a & 1276 \\
\hline 9 & miR-200c & 1370 & miR-29a & 1322 & miR-320 & 1367 & miR-24 & 1199 \\
\hline 10 & let-7c & 1145 & let-7b & 1131 & miR-17-5p & 1326 & miR-494 & 1042 \\
\hline 11 & miR-16 & 1051 & miR-221 & 1125 & miR-103 & 1171 & miR-221 & 1010 \\
\hline 12 & miR-222 & 1010 & miR-200c & 1089 & miR-107 & 1047 & miR-200c & 1002 \\
\hline
\end{tabular}

Each value is shown in net intensity after quantile normalization.

Table II. Differential expression of miRNAs in malignant and benign squamous epithelial cell lines by statistical analysis of miRNA microarray.

6 HNSCC cell lines vs. HOK 7 ESCC cell lines vs. HEEC

\begin{tabular}{|c|c|c|c|c|c|c|c|c|}
\hline \multicolumn{9}{|c|}{ Up-regulated } \\
\hline 1 & miR-30a-5p & 612 & 243 & $(2.50)$ & miR-16 & 2742 & 373 & (7.29) \\
\hline 2 & miR-30d & 693 & 291 & $(2.28)$ & $\operatorname{miR}-15 b$ & 918 & 198 & (4.57) \\
\hline 3 & miR-24 & 2082 & 964 & (2.13) & miR-30a-5p & 983 & 243 & (4.03) \\
\hline 4 & miR-21 & 3552 & 2054 & (1.69) & miR-17-5p & 1326 & 359 & $(3.65)$ \\
\hline 5 & $\operatorname{miR}-27 b$ & 554 & 308 & (1.64) & $\operatorname{miR}-320$ & 1367 & 383 & (3.57) \\
\hline 6 & miR-16 & 1051 & 673 & (1.56) & miR-106a & 1042 & 371 & (2.81) \\
\hline 7 & miR-103 & 774 & 493 & $(1.55)$ & $\operatorname{miR}-200 \mathrm{c}$ & 2219 & 1002 & $(2.20)$ \\
\hline 8 & $\operatorname{miR}-342$ & 1393 & 149 & $(1.51)$ & $\operatorname{miR}-103$ & 1171 & 601 & (1.91) \\
\hline 9 & miR-107 & 707 & 466 & $(1.50)$ & miR-107 & 1047 & 588 & $(1.76)$ \\
\hline 10 & miR-26a & 618 & 400 & $(1.45)$ & miR-21 & 4173 & 2851 & $(1.45)$ \\
\hline \multicolumn{9}{|c|}{ Down-regulated } \\
\hline 1 & $\operatorname{miR}-125 b$ & 178 & 971 & $(0.18)$ & miR-31 & 915 & 4366 & $(0.21)$ \\
\hline 2 & let-7f & 251 & 773 & $(0.32)$ & miR-203 & 207 & 834 & $(0.24)$ \\
\hline 3 & miR-106a & 289 & 699 & $(0.41)$ & miR-29a & 464 & 1276 & $(0.36)$ \\
\hline 4 & miR-92 & 197 & 446 & $(0.44)$ & let-7c & 608 & 1559 & (0.38) \\
\hline 5 & miR-494 & 367 & 805 & $(0.45)$ & miR-221 & 501 & 1010 & $(0.49)$ \\
\hline
\end{tabular}

Each value is shown in net intensity after quantile normalization. Brackets indicate fold increases compared to the intensity in HOK or HEEC.

\section{Results}

Expression of miRNAs in sqaumous epithelial cell lines by microarray analysis. For miRNA microarray analysis of human HNSCC and ESCC, we prepared 4 sets of miRNA extracts. The HNSCC set consisted of a mixture of equal amounts of miRNAs derived from 6 tongue SCC cell lines (SCC4, SCC9, SCC15, SCC25, OSC19 and OSC20), while the ESCC set consisted of those from 7 ESCC cell lines (KYSE30, KYSE70, KYSE140, KYSE170, KYSE180, KYSE220 and KYSE270). Human normal oral or esophageal squamous epithelial cell set consisted only of HOK or HEEC cell line, respectively. Each microarray assay was performed three times using different sets of miRNA extracts, and the results were averaged and normalized with inner controls. The 12 most highly expressed miRNAs in 4 sets of microarrays are listed in Table I. Net intensities of miR-205 showed highest expression in all 4 sets examined, and those of miR-21 showed second highest expression in HNSCC and ESCC sets and third highest expression in normal squamous epithelial sets. Four miRNAs let-7a, miR-23a, miR-23b and miR-200c were also shown to be expressed abundantly in all 4 sets. We also 
Table III. Patient profiles, location and histological differentiation of FFPE tissue samples used in this study.

\begin{tabular}{|c|c|c|c|c|}
\hline Case & Age & Gender & Location & Differentiation \\
\hline 1 & 65 & Male & Tongue & Well \\
\hline 2 & 58 & Male & Tongue & Well \\
\hline 3 & 76 & Male & Tongue & Well to moderate \\
\hline 4 & 50 & Male & Tongue & Well \\
\hline 5 & 78 & Female & Tongue & Well \\
\hline 6 & 76 & Male & Tongue & Well to moderate \\
\hline 7 & 47 & Female & Tongue & Well \\
\hline 8 & 70 & Male & Tongue & Well \\
\hline 9 & 54 & Male & Tongue & Well to moderate \\
\hline 10 & 36 & Male & Tongue & Well \\
\hline 11 & 70 & Female & Tongue & Well \\
\hline 12 & 61 & Male & Tongue & Well \\
\hline 13 & 73 & Female & Tongue & Well \\
\hline 14 & 54 & Male & Esophagus & Moderate \\
\hline 15 & 69 & Male & Esophagus & Well to moderate \\
\hline 16 & 62 & Male & Esophagus & Well to moderate \\
\hline 17 & 50 & Male & Esophagus & Moderate \\
\hline 18 & 78 & Male & Esophagus & Moderate to poor \\
\hline 19 & 76 & Male & Esophagus & Well to moderate \\
\hline 20 & 80 & Male & Esophagus & Moderate to poor \\
\hline 21 & 76 & Male & Esophagus & Well to moderate \\
\hline 22 & 76 & Male & Esophagus & Well to moderate \\
\hline 23 & 79 & Male & Esophagus & Well to moderate \\
\hline 24 & 53 & Female & Skin & Well \\
\hline 25 & 82 & Male & Skin & Well \\
\hline 26 & 67 & Male & Skin & Well \\
\hline 27 & 81 & Male & Skin & Well \\
\hline 28 & 86 & Male & Skin & Well \\
\hline 29 & 60 & Female & Cervix & Poor \\
\hline 30 & 71 & Female & Cervix & Well \\
\hline 31 & 63 & Female & Cervix & Well \\
\hline 32 & 71 & Female & Cervix & Moderate \\
\hline 33 & 38 & Female & Cervix & Well \\
\hline 34 & 78 & Male & Lung & Poor \\
\hline 35 & 83 & Male & Lung & Moderate \\
\hline 36 & 81 & Male & Lung & Moderate \\
\hline 37 & 74 & Female & Lung & Poor \\
\hline 38 & 63 & Female & Lung & Well \\
\hline 39 & 71 & Male & Lung & Mixed \\
\hline 40 & 58 & Male & Lung & Mixed (well to moderate) \\
\hline 41 & 64 & Female & Lung & Mixed \\
\hline 42 & 76 & Female & Lung & Mixed (well to moderate) \\
\hline 43 & 73 & Male & Lung & Mixed \\
\hline 44 & 77 & Male & Colon & Moderate \\
\hline 45 & 76 & Male & Colon & Well \\
\hline 46 & 75 & Male & Colon & Well \\
\hline 47 & 69 & Male & Colon & Moderate \\
\hline 48 & 87 & Female & Colon & Moderate \\
\hline
\end{tabular}




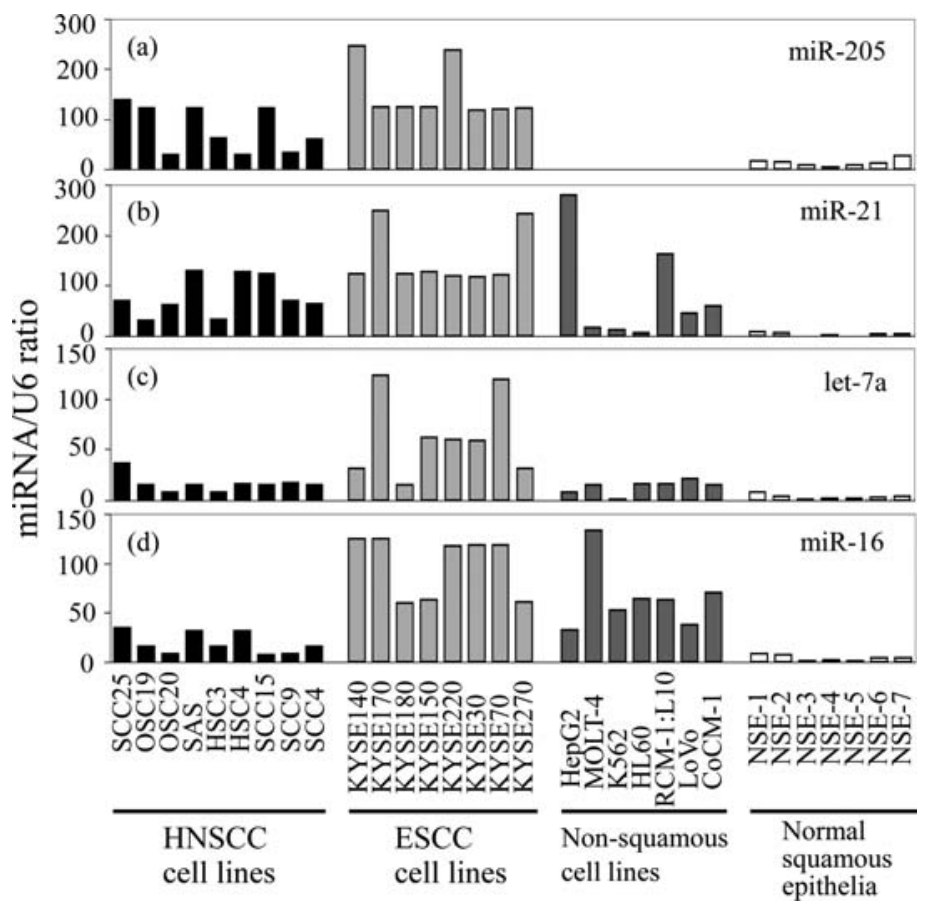

Figure 1. Expression of miR-205 (a), miR-21 (b), let-7a (c) and miR-16 (d) in various cancer cell lines and normal oral squamous epithelia obtained from frozen sections by LCM. Note that consistent high expression (net intensity) of miR-205 (a) was observed in all HNSCC and ESCC cell lines as well as normal squamous epithelia (NSE), whereas no detectable level of the expression was observed in non-sqaumous epithelial cell lines (1 hepatocellular, 3 hematological and 3 colorectal cancer cell lines). MiR-21 (b), let-7a (c) and miR-16 (d) were also highly expressed and up-regulated in HNSCC and ESCC cell lines compared to normal squamous epithelia (NSE).

examined differentially expressed miRNAs between benign and malignant squamous epithelial sets. Significantly up- or down-regulated miRNAs in HNSCC and ESCC sets compared to corresponding normal squamous epithelial sets are listed in Table II. MiR-30a-5p, miR-21, miR-16 and miR107 were up-regulated in both HNSCC and ESCC sets, while no miRNAs were commonly down-regulated in these sets. Among these highly and/or differentially expressed miRNAs, we selected miR-205, miR-21, let-7a and miR-16 in the next step of validation study, because former four miRNAs showed not only up-regulation but also high level expression both in HNSCC and ESCC, and their functions including target mRNAs have been widely studied in malignancies of other organs (6-9). MiR-30a-5p was also subjected to the validation study, although its specific expression or function remains unclear.

Validation of miRNA expression by real-time $q R T-P C R$. In order to validate the miRNA expression profiles of HNSCC and ESCC by microarray analysis, we performed real-time qRT-PCR for various cancer cell lines and FFPE tissue samples. Patient profiles, location, histological differentiation of FFPE tissue samples are summarized in Table III. First, we examined relative expression of miR-205, miR-21, let-7a and miR-16 in 9 tongue SCC cell lines, 8 ESCC cell lines, 1 hepatocellular carcinoma cell line, 3 hematological cancer cell lines, 3 colorectal adenocarcinoma cell lines, and 7 normal oral squamous epithelia from frozen sections obtained by LCM. As shown in Fig. 1, consistent high expression (net intensity) of miR-205 was observed in all SCC cell lines as well as normal squamous epithelia, whereas no detectable level of the expression was observed in non-sqaumous
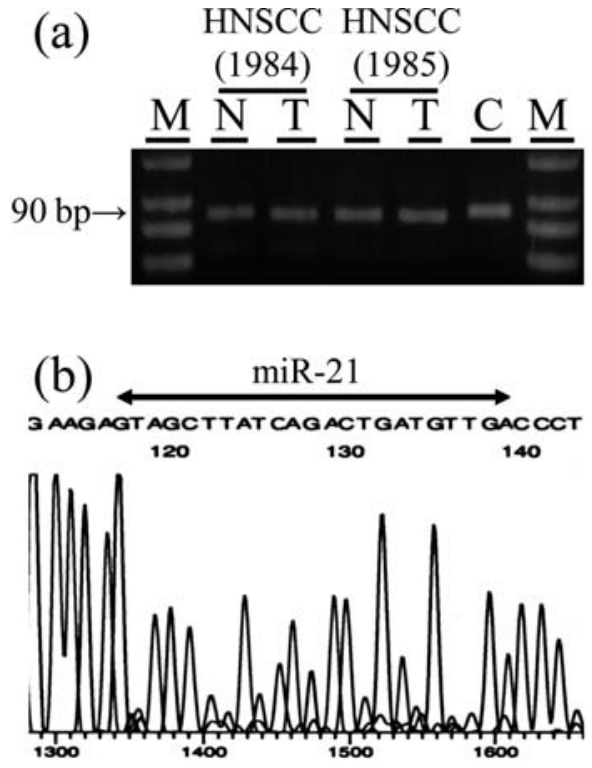

Figure 2. RT-PCR analysis (a) following nucleotide sequencing (b) of miR21 extracted from FFPE tissue samples stored over 20 years. M, molecular marker; N, normal squamous epithelial tissue; T, SCC tissue; $\mathrm{C}$, positive control human heart total RNA (target, miR-24). The arrow indicates the nucleotide sequence of miR-21.

epithelial cell lines ( 1 hepatocellular, 3 hematological and 3 colorectal cancer cell lines). MiR-21, let-7a and miR-16 were also highly expressed and up-regulated in SCC cell lines compared to normal squamous epithelia $(\mathrm{p}<0.05)$. These results suggested that miR-205 could be a specific marker of squamous epithelium, and miR-21, let-7a and miR-16 could 
A

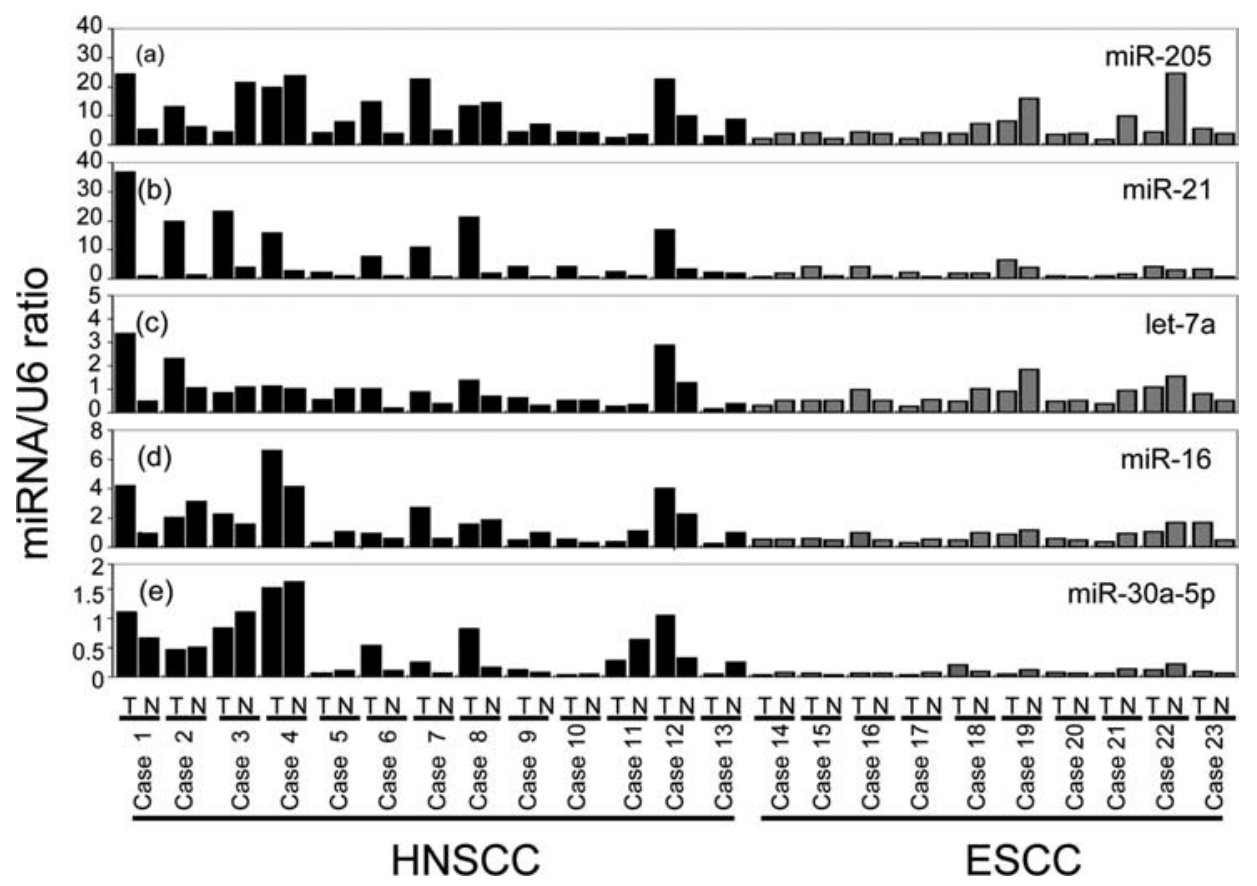

B

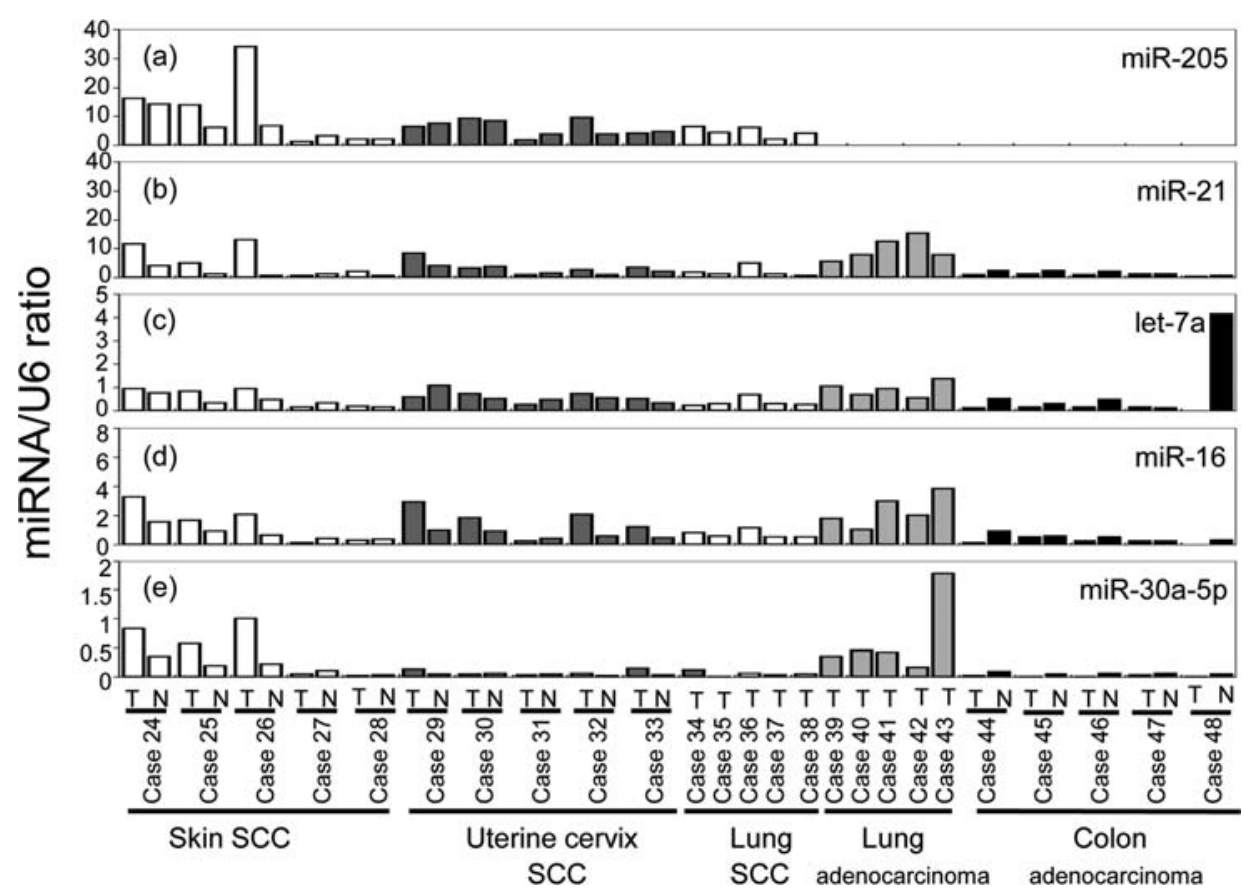

Figure 3. Expression of miR-205 (a), miR-21 (b), let-7a (c), miR-16 (d) and miR-30a-5p (e) in HNSCC and ESCC tissues with paired normal squamous epithelia obtained from FFPE tissue samples by LCM. Note that high expression (net intensity) of miR-205 (a) was observed in all HNSCC, ESCC and SCC tissue samples of other organs regardless of normal and malignant squamous epithelial cells, whereas no detectable level of the expression was observed in normal or malignant lung or colorectal epithelial cells. High expression and significant up-regulation of miR-21 (b) was also observed in HNSCC and ESCC tissues compared to the paired normal squamous epithelia.

be up-regulated in HNSCC and ESCC. In further validation, we next examined the expression of these 4 miRNAs and miR-30a-5p in clinical samples. In preliminary experiments, we confirmed that high-quality miRNAs detectable in RT-PCR were extracted from FFPE tissues stored over 20 years and the nucleotide sequences of amplified miRNAs were correct (Fig. 2). MiRNAs from carcinoma cells and paired normal epithelial cells were separately extracted by LCM from FFPE tissue samples of 13 HNSCC, 10 ESCC, 5 skin SCC, 5 cervical SCC, 5 lung SCC, 5 lung adenocarcinoma and 5 colorectal adenocarcinoma patients. High expression (net intensity) of miR-205 was also observed in all HNSCC, ESCC, skin SCC, cervical SCC, 5 lung SCC tissue samples regardless of normal and malignant squamous epithelial cells, whereas no detectable level of the expression was observed in lung adenocarcinoma and normal or malignant colorectal epithelial cells (Fig. 3). This confirmed miR-205 as a specific marker miRNA of squamous epithelium. Significant upregulation of miR-21 was also observed in HNSCC and ESCC tissues compared to the paired normal squamous epithelia 
(a)

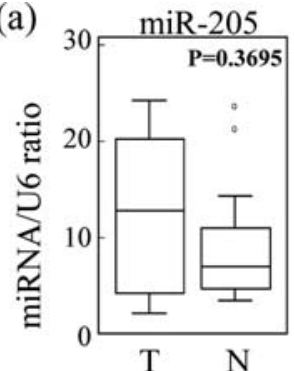

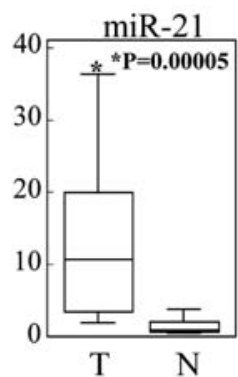
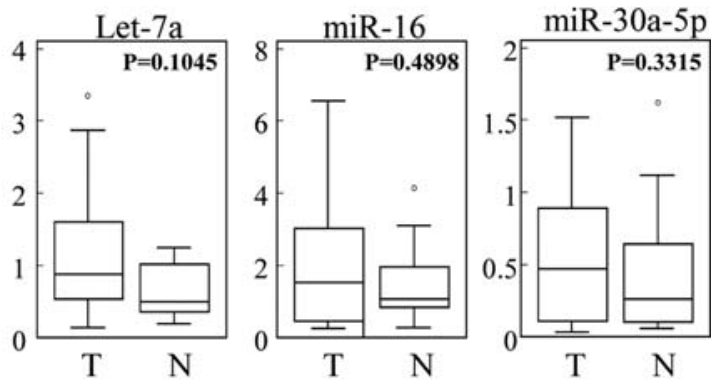

(b)
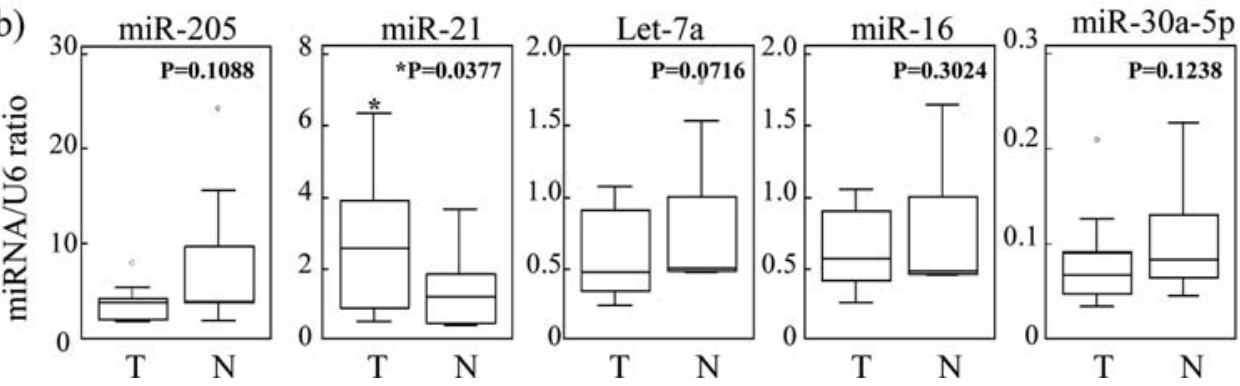

Figure 4. Differential expression of miR-205, miR-21, let-7a, miR-16 and miR-30a-5p in HNSCC (a) and ESCC (b) tissues compared to paired normal squamous epithelia. Significant up-regulation of miR-21 but not miR-205, let-7a, miR-16, nor miR-30a-5p was observed in HNSCC and ESCC tissues, compared to the paired normal squamous epithelia.

(Figs. 3 and 4). The average expression of miR-21 in HNSCC and ESCC tissues was 12.70 and 2.77 (SE=2.91 and $0.59)$ compared to the paired normal squamous epithelial cells, which was 1.51 and $1.51(\mathrm{SE}=0.30$ and 0.35$)$ ( $p=0.00005$ and 0.0377) (Fig. 4). However, no statistically significant up-regulation of let-7a, miR-16 and miR-30a-5p was observed in HNSCC and ESCC tissue samples [ $\mathrm{p}=0.1045,0.4898$ or 0.3315 (HNSCC) and $\mathrm{p}=0.0716$, 0.3024 or 0.1238 (ESCC), respectively], although let-7a and miR-16 were statistically up-regulated in HNSCC and ESCC cell lines compared to the normal squamous epithelia derived from frozen sections by LCM (Fig. 1). Since let-7a and miR-16 have been widely shown to function as tumorsuppressive miRNAs in B-cell lymphomas and colonic and lung cancers by controlling the translation of BCL2 and RAS oncogenes, respectively (6-9), our results suggested that miRNA expression and their target mRNAs of HNSCC and ESCC were quite different from those of other organ malignancies. Although miR-21 would be a putative oncogenic miRNA in HNSCC and ESCC similarly with other cancers, let-7a and miR-16 might not be a tumor suppressive miRNA in these SCC.

\section{Discussion}

MiRNAs are a new class of non-coding small RNAs that regulate cell proliferation and various cellular functions through interfering the translation of target mRNAs $(4,5)$. Recent studies have demonstrated that altered expression of several miRNA induces various human malignancies (6-9). The expression profiles have been established for many different cancers and seem to be unique to each cancer (6-9). However, scarce data exist on the role of miRNAs in HNSCC and ESCC. In this study, we investigated the
miRNA expression of malignant and normal squamous epithelia of oral cavity, the esophagus and other organs. The results revealed that miR-205 was highly expressed in both benign and malignant squamous epithelia, resulting in a potential specific marker miRNA of squamous epithelia. Also, we found that miR-21 might be an oncogenic miRNA similarlyto other organ malignancies, but miR-16 and let-7a might not function as tumor-suppressive miRNAs in HNSCC and ESCC differently from malignancies of other organs.

It has been recently reported that miR-205 was highly expressed in cell lines of HNSCC and ESCC compared to those of other organ malignancies $(1,2,11)$. Fletcher et al reported that miR-205 was abundantly expressed in mouse tissues involving squamous epithelium as well as in HNSCC cell lines (12). In this study, we have confirmed that miR-205 showed the highest expression in both benign and malignant squamous epithelial cell lines as well as in both epithelial cells obtained from FFPE tissue samples by LCM. Its function including target mRNAs in squamous epithelium has not yet been elucidated, but it might regulate epithelial to mesenchymal transition by targeting ZEB1 and SIP1 in collaboration with miR-200 family miRNAs (13) and target the HER3 oncogene in human breast cancer (14). Lipid phosphatase SHIP2 was reported to be one of the targets of miR-205 in corneal epithelium and the corneal epithelialspecific miR-184 could interfere with the ability of miR-205 to suppress SHIP2 levels (15). It has been reported that lowlevel expression of miR-205 is a prognostic marker of HNSCC (16). Since the present study demonstrated that miR-205 was specifically expressed in squamous epithelium but not in other types of epithelia, miR-205 might become a specific biomarker to distinguish SCC from other carcinomas. This might be useful to determine the cell origin of poorly differentiated or undifferentiated carcinomas as well as to 
locate the primary site of metastatic carcinomas. In addition, Fletcher et al reported that miR-205 could be used for wholelymph node processing and qRT-PCR analysis to detect micrometastasis of SCC (12). Although no apparent alteration was observed in the expression of miR-205 between normal and malignant squamous epithelium in this study, its consistent expression makes it reliable surrogate marker for the presence of squamous epithelium.

Of interest in our study is that miRNAs were successfully extracted from FFPE tissue samples and their application for histopathological diagnosis was confirmed, in addition to routine hematoxylin-eosin and immunohistochemical staining methods. MiRNAs are stable small molecules and not degraded even under heat- or acid-degenerative conditions (5). Indeed, we could successfully extract abundant high-quality miRNAs detectable by RT-PCR from FFPE tissues stored at room temperature over 20 years (Fig. 2). Thus, miRNAs took specific advantage compared to their target mRNAs, which are unstable and mostly degraded in FFPE tissue samples. However, there are only a few studies concerning the use of miRNA for molecular diagnosis in the histopathological fields. Our present study clearly demonstrated the usage of miR-205 as a squamous epithelial-specific marker in routinely processed FFPE tissue samples for histopathological diagnosis. If various specific miRNA markers are identified after this, molecular diagnosis using miRNA in routine FFPE tissue samples would come into wide use in most pathological laboratories, instead of molecular diagnosis using mRNA in frozen tissue samples.

Mir-21 has been investigated widely in various human malignancies including hematological malignancies and glioblastomas as a putative oncogenic miRNA $(6-9,17,18)$. It has been also implicated in 5-FU resistance (19), hypertrophic heart muscles (20) and vascular formation (21). MiR-21 has been shown to act as an oncogenic miRNA by targeting tumor suppressor gene tropomyosin 1 (TPMI) (22). Chan et al reported that miR-21 functions as an anti-apoptotic agent through activation of caspase by knockdown of miR-21 (18). It has been shown that miR-21 was involved in invasion and metastasis (23), modulation of matrix metalloproteinase-2 and matrix metalloproteinase-9 (24) and regulation of STAT3mediated pathway (25). In the present study, miR-21 was also markedly up-regulated in HNSCC and ESCC tissues compared to paired normal squamous epithelia. Target mRNA of miR-21 in HNSCC and ESCC was not examined in this study, but it is likely that miR-21 inhibits several mRNAs leading to a cascade of events that prevent apoptosis and increase cellular proliferation.

Let-7a and miR-16 have also been widely studied as tumor-suppressive miRNAs. Let-7 family miRNAs including let-7a are down-regulated in various human malignancies and are shown to negatively regulate $R A S$ oncogenes frequently activated in many cancers $(6-9,26)$. Down-regulation of let-7 family miRNAs is also shown to cause radioresistance and results in poor prognosis (27). RAS oncogenes are frequently overexpressed without mutation in HNSCC and ESCC (28). On the other hand, the expression of miR-16 induces apoptosis by targeting anti-apoptotic gene BCL2 and is down-regulated in many human malignancies including hematological cancer $(6-9,29,30)$. However, in the present study, the expression of let-7a and miR-16 was not down-regulated and even tended to be up-regulated although without statistical significance in HNSCC. Tran et al also reported high expression of let-7a and miR-16 in several HNSCC cell lines (2). The reason is unknown, but we assume that target mRNAs of these miRNAs might be different between SCC and other cancers such as adenocarcinomas and lymphoma/leukemia and might not be involved in the initiation and progression of HNSCC. Also, miR-30a-5p is not statistically altered in the validation study using clinical samples, although it is apparently up-regulated in the initial miRNA microarray using cell lines. This discrepancy might be due to the different cell sources used, in addition to unknown functions of this miRNA.

Several miRNAs other than those above mentioned have been reported to be involved in HNSCC and ESCC. It has been reported that high expression of mir-103/107 correlated with poor survival in ESCC (31). MiR-184 has also been reported to act as a potential oncogenic miRNA in HNSCC (32). However, miR-103/107 was not statistically altered between benign and malignant squamous epithelial cell lines, although these miRNAs showed relatively high expression both in benign and malignant squamous epithelial cell lines in our microarray analysis. MiR-184 showed low expression with no drastic alteration between benign and malignant squamous epithelia. Also, miR-133a, miR-133b, miR-137, miR-193a and miR-494 were less expressed in both benign and malignant squamous epithelial cell lines, although they have been reported to be down-regulated in $\operatorname{HNSCC}(2,3$, 32,33).

In summary, we examined the expression of miRNAs in HNSCC and ESCC and found two possible marker miRNAs in these cancers. MiR-205 might be a specific marker miRNA of both normal and malignant squamous epithelia, while miR-21 might be a putative oncogenic miRNA in HNSCC and ESCC. Further study for their use as histopathological diagnostic, prognostic or therapeutic marker is now undergoing in our laboratory.

\section{Acknowledgements}

The authors wish to thank Mr. H. Kato and Mrs. T. Ozaki for their skillful technical assistance. This study was supported in part by Grant-in-Aid for Scientific Research (C) No.20590382 and Grant-in-Aid for Young Scientist (B) No.21790382 from the Ministry of Education, Science, Sports and Culture, Japan.

\section{References}

1. Feber A, Xi L, Luketich JD, et al: MicroRNA expression profiles of esophageal cancer. J Thorac Cardiovasc Surg 135: 255-260, 2008.

2. Tran N, McLean T, Zhang X, et al: MicroRNA expression profiles in head and neck cancer cell lines. Biochem Biophys Res Commun 358: 12-17, 2007.

3. Chang SS, Jiang WW, Smith I, et al: MicroRNA alterations in head and neck squamous cell carcinoma. Int J Cancer 123: 2791-2797, 2008.

4. Lagos-Quintana, Rauhut R, Lendeckel T and Tuschl T: Identification of novel genes coding for small expressed RNAs. Science 294: 853-858, 2001.

5. Bartel DP: MicroRNAs: genomics, biogenesis, mechanism and function. Cell 116: 281-297, 2004.

6. Zhang B, Pan X, Cobb GP and Anderson TA: MicroRNAs as oncogenes and tumor suppressors. Dev Biol 289: 3-16, 2006. 
7. Esquela-Kerscher A and Slack FJ: Oncomirs-microRNAs with a role in cancer. Nat Rev Cancer 6: 259-269, 2006.

8. Tamaru Y and Hayashizaki Y: Cancer research with non-coding RNA. Cancer Sci 97: 1285-1290, 2006.

9. Lu J, Getz G, Miska EA, et al: MicroRNA expression profiles classify human cancers. Nature 435: 834-838, 2005.

10. Calin GA and Croce CM: MicroRNA signatures in human cancers. Nat Rev Cancer 6: 857-866, 2006.

11. Jiang J, Lee EJ, Gusev Y and Schmittgen TD: Real-time expression profiling of microRNA precursors in human cancer cell lines. Nucleic Acids Res 33: 5394-5403, 2005.

12. Fletcher AM, Heaford AC and Trask DK: Detection of metastatic head and neck squamous cell carcinoma using the relative expression of tissue-specific mir-205. Transl Oncol 1: 202-208, 2008.

13. Gregory PA, Bert AG, Paterson EL, et al: The miR-200 family and miR-205 regulate epithelial to mesenchymal transition by targeting ZEB1 and SIP1. Nat Cell Biol 10: 593-601, 2008.

14. Iorio MV, Casalini P, Piovan C, et al: microRNA-205 regulates HER3 in human breast cancer. Cancer Res 69: 2195-2200, 2009.

15. Yu J, Ryan DG, Getsios S, Oliveira-Fernandes M, Fatima A and Lavker RM: MicroRNA-184 antagonizes microRNA-205 to maintain SHIP levels in epithelia. Proc Natl Acad Sci USA 105: 19300-19305, 2008.

16. Childs G, Fazzari M, Kung G, et al: Low-level expression of microRNAs let-7d and miR-205 are prognostic markers of head and neck squamous cell carcinoma. Am J Pathol 174: 736-745, 2009.

17. Calin GA, Liu CG, Sevignani C, et al: MicroRNA profiling reveals distinct signatures in B cell chronic lymphocytic leukemias. Proc Natl Acad Sci USA 101: 11755-11760, 2004

18. Chan JA, Krichevsky AM and Kosik KS: MicroRNA-21 is an antiapoptotic factor in human glioblastoma cells. Cancer Res 65: 6029-6033, 2005

19. Rossi L, Bonmassar E and Faraoni I: Modification of miR gene expression pattern in human colon cancer cells following exposure to 5-fluorouracil in vitro. Pharmacol Res 56: 248-253, 2007.

20. Cheng Y, Ji R, Yue J, et al: MicroRNAs are aberrantly expressed in hypertrophic heart: do they play a role in cardiac hypertrophy? Am J Pathol 170: 1831-1840, 2007.

21. Ji R, Cheng Y, Yue J, et al: MicroRNA expression signature and antisense-mediated depletion reveal an essential role of microRNA in vascular neointimal lesion formation. Circ Res 100: 1579-1588, 2007
22. Zhu S, Si ML, Wu H and Mo YY: MicroRNA-21 targets the tumor suppressor gene tropomyosin 1 (TPM1). J Biol Chem 282: 14328-14336, 2007.

23. Zhu S, Wu H, Wu F, Nie D, Sheng S and Mo YY: MicroRNA-21 targets tumor suppressor genes in invasion and metastasis. Cell Res 18: 350-359, 2008.

24. Meng F, Henson R, Wehbe-Janek H, Ghoshal K, Jacob ST and Patel T: MicroRNA-21 regulates expression of the PTEN tumor suppressor gene in human hepatocellular cancer. Gastroenterology 133: 647-658, 2007.

25. Loffer D, Brocke-Heidrich K, Pfeifer G, et al: Interleukin-6dependent survival of multiple myeloma cells involves the Stat3-mediated induction of microRNA-21 through a highly conserved enhancer. Blood 110: 1330-1333, 2007.

26. Johnson SM, Grosshans H, Shingara J, et al: RAS is regulated by the let-7 microRNA family. Cell 120: 635-647, 2005.

27. Weidhaas JB, Babar I, Nallur SM, et al: MicroRNA as potential agents to alter resistance to cytotoxic anticancer therapy. Cancer Res 67: 11111-11116, 2007.

28. Hoa M, Davis SL, Ames SJ and Spanjaard RA: Amplification of wild-type K-ras promotes growth of head and neck squamous cell carcinoma. Cancer Res 62: 7154-7156, 2002.

29. Calin GA, Dumitru CD, Shimizu M, et al: Frequent deletions and down-regulation of micro-RNA genes miR15 and miR16 at $13 q 14$ in chronic lymphocytic leukemia. Proc Natl Acad Sci USA 99: 15524-15529, 2002.

30. Cimmino A, Calin GA, Fabbri M, et al: miR-15 and miR-16 induce apoptosis by targeting BCL2. Proc Natl Acad Sci USA 102: 13944-13949, 2005.

31. Guo Y, Chen Z, Zhang L, et al: Distinctive microRNA profiles relating to patient survival in esophageal squamous cell carcinoma. Cancer Res 68: 26-34, 2008.

32. Wong TS, Liu XB, Wong BY-H, Ng RW-M, Yuen AP-W and Wei WI: Mature miR-184 as potential oncogenic microRNA of squamous cell carcinoma of tongue. Clin Cancer Res 14: 2588-2592, 2008

33. Wong TS, Liu XB, Ho AC-W, Yuen AP-W, Ng RW-M and Wei WI: Identification of pyruvate kinase type M2 as potential oncoprotein in squamous cell carcinoma of tongue through microRNA profiling. Int J Cancer 123: 251-257, 2008. 